LBL-36460

\title{
Design and Construction of a Large Aperture, Quadrupole Electromagnet Prototype for ILSE
}

\author{
M. Stuart, A. Faltens, W.M. Fawley, C. Peters, and M.C. Vella \\ Accelerator and Fusion Research Division \\ Lawrence Berkeley Laboratory \\ University of California \\ Berkeley, California 94720
}

April 1995

\section{DISCLAIMER}

This report was prepared as an account of work sponsored by an agency of the United States Government. Neither the United States Government nor any agency thereof, nor any of their employees, makes any warranty, express or implied, or assumes any legal liability or responsibility for the accuracy, completeness, or usefulness of any information, apparatus, product, or process disclosed, or represents that its use would not infringe privately owned rights. Reference herein to any specific commercial product, process, or service by trade name, trademark, manufacturer, or otherwise does not necessarily constitute or imply its endorsement, recommendation, or favoring by the United States Government or any agency thereof. The views and opinions of authors expressed herein do not necessarily state or reflect those of the United States Government or any agency thereof.

This work was supported by the Director, Office of Energy Research, Office of High Energy Physics, of the U.S. Department of Energy under Contract No. DE-AC03-76SF00098. 


\section{DISCLAIMER}

Portions of this document may be illegible in electronic image products. Images are produced from the best available original document. 


\title{
DESIGN AND CONSTRUCTION OF A LARGE APERTURE, QUADRUPOLE ELECTROMAGNET PROTOTYPE FOR ILSE*
}

\author{
M. Stuart, A. Faltens, W.M. Fawley, C. Peters, and M.C. Vella \\ Lawrence Berkeley Laboratory, University of California \\ Berkeley, CA 94720 USA
}

Abstract

We are currently constructing a prototype quadrupole electromagnet for the proposed Induction Linac Systems Experiment (ILSE) at LBL. ILSE will address many physics and engineering issues relevant to the design of a heavyion fusion driver accelerator. The pulsed electromagnet has two layers of current windings and will produce a field gradient exceeding $25 \mathrm{~T} / \mathrm{m}$ at a repetition rate of $1 \mathrm{~Hz}$ steady-state. In this paper, we discuss how the interaction of various concerns such as maximum dynamic aperture, short lattice period, field quality, iron yoke weight, heat transfer, and voltage standoff have led to our particular design choices. We also present 2- and 3-D numerical calculations concerning field topography and the results of transport simulations of space-charge dominated ion beams with ILSE parameters.

\section{Introduction}

The great bulk of the beam transport in heavy ion fusion (HIF) drivers will be done with strong-focusing, magnetic quadrupoles. In many driver designs, electrostatic quadrupoles will provide focusing from the $\sim 2 \mathrm{MV}$ injector to approximately $50-100 \mathrm{MV}$ at which the transition to magnetic transport is made. The actual transition energy depends critically upon the maximum transportable current by the magnetic quadrupole lattice which scales as $(a / L)^{2} V^{3 / 2}$ where $a$ is the useful dynamic aperture, $L$ is the half-lattice period, and $V$ is the beam energy. Thus, in the low energy portion of the magnetic transport section, there is a premium for maximizing the aperture ratio.

In the past few years, the LBL HIF group has developed a conceptual design for the 10-MV ILSE ("Induction Systems Linac Experiment") accelerator which, if and when fully funded by a presently parsimonious DOE, will allow examination of many physics and engineering issues common to HIF drivers. As part of this effort, we have designed and are currently building the latest iteration of a large aperture, short lattice period, pulsed electromagnet prototype to be used in the 5-10 MV magnetic transport section of ILSE. The magnet must operate dependably at a continuous, $1-\mathrm{Hz}$ repetition rate and transport $\approx 1-1.5 \mu \mathrm{C} / \mathrm{m}$ of a space-charge dominated $\mathrm{K}^{+1}$ beam with minimal emittance degradation. In the remainder of this paper, we discuss the design philosophy and tradeoffs that have determined our particular choices for this magnet.

\footnotetext{
-This work was supported by the Director, Office of Energy Research, Office of Fusion Energy, U.S. Departinent of Energy, under Contract No. DE-AC03-76SF00098
}

\section{Basic Beam and Magnet Parameters}

As presently conceived, the ILSE injector will produce four individual beams of $\sim 0.2 \mu \mathrm{C} / \mathrm{m}$ line charge density which will be accelerated by induction cores, slowly compressed longitudinally to perhaps as much as $0.3 \mu \mathrm{C} / \mathrm{m}$, and transported via electrostatic quadrupoles up to $\approx 5 \mathrm{MV}$ energy. At this point, the beams will be combined and merged into one single beam and then transported magnetically to the beam dump. The half-lattice period $L$ increases in the electrostatic transport region from $0.25 \mathrm{~m}$. to $0.40 \mathrm{~m}$ and the present beam combiner/merger design has $L \approx 0.5 \mathrm{~m}$.

For a strongly space-charge dominated beam $\left(\sigma \ll \sigma_{0}\right)$,

$$
\left(\frac{a_{b}}{L}\right)^{2}=\frac{2 Q}{\left(1-\cos \sigma_{0}\right)}=\frac{\lambda}{4 \pi \epsilon_{0} V}
$$

where $Q$ is the beam perveance, and $\sigma$ and $\sigma_{0}$ are respectively the undepressed and space-charge depressed phase advance per full lattice period $2 L$. If $\sigma_{0} \approx 72^{\circ}$ at $5 \mathrm{MV}$ and $\lambda=1.5 \mu \mathrm{C} / \mathrm{m}$, the perveance is $2.7 \times 10^{-3}$ and the required $a_{b} / L$ is less than 0.1. Although this seems to be a comfortably small number, in fact due to longitudinal "packing factor" constraints induced by the space required for the accelerating gap, insulators, couplers, etc., the magnet windings in the latest ILSE prototype design occupy only about one-half of the half-lattice period. Hence, the ratio of the winding radius to the effective length exceeds 0.2 and one must be careful to minimize anharmonic fringe field and higher order multipole components.

The required field gradient may be roughly estimated from the relation

$$
\sigma_{\circ}=\frac{v_{z} B^{\prime}}{V} \frac{L^{2}}{2 \pi}
$$

where $B^{\prime}$ is measured in $\mathrm{T} / \mathrm{m}$ and we presume that the field is dominated by the fundamental longitudinal harmonic. For $5 \mathrm{MV} \mathrm{K}{ }^{+1}, L=0.5 \mathrm{~m}$ and $\sigma_{0}=72^{\circ}$, the required gradient is about $18 \mathrm{~T} / \mathrm{m}$.

\section{A. Current Winding Geometry}

To minimize anharmonic field components, we follow the philosophy of Laslett et al.[1] and make such components disappear in the $z$-integrated sense over a half-lattice period. Ignoring for the moment turn-to-turn crossovers, Our actual coil ends have elliptical projections in $(\theta, z)$ space (see Fig. 1 of ref. [2]), a geometry that avoids sharp bends for the individual wires. In order to limit the current per turn to $5 \mathrm{kA}$ or less, the magnet has two layers of twelve turns each. Ideally, the end curve of each turn would be individually separated in $z$ from its neighbors (this allows 


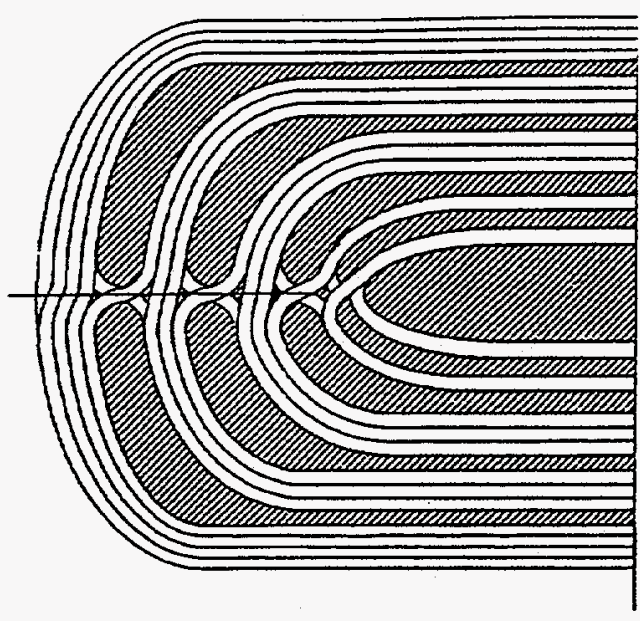

Figure. 1. Artist's depiction of the projected coil turn geometry of the quadrupole magnet prototype.

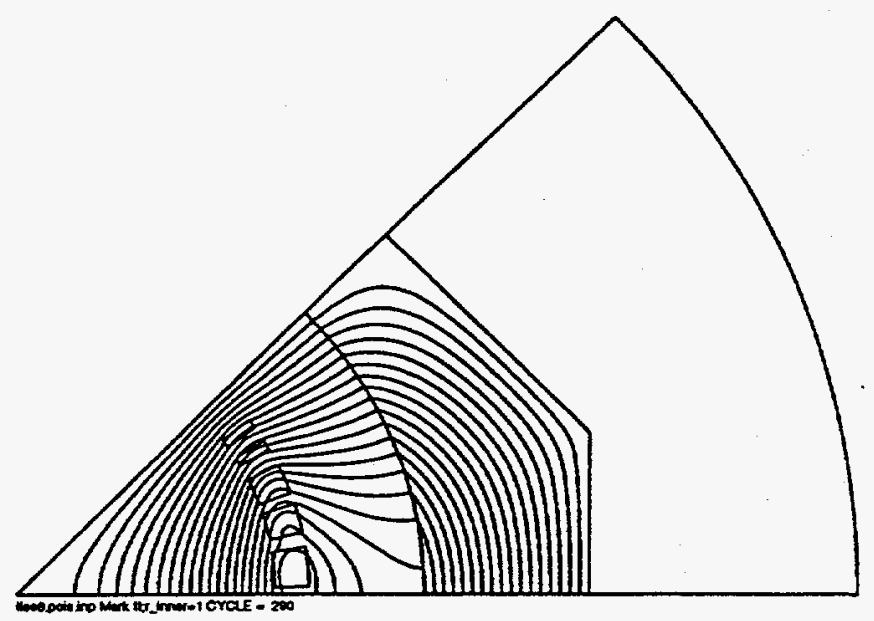

Figure. 2. 2D POISSON results showing the field line topology in the $r-\theta$ plane of an octant of the quadrupole magnet plus iron yoke. The midplane field gradient at $r=$ 0 was $26.5 \mathrm{~T} / \mathrm{m}$. The peak field in the iron yoke is $1.5 \mathrm{~T} / \mathrm{m}$.

one to suppress the maximum number of unwanted harmonic overtones in $\theta$ ) but in practice we found it necessary to distribute the 12 turns in each layer into 5 individual "blocks" in a 4-3-3-1-1 pattern (see Fig. 1) to obtain sufficient spacing for the cutting tool in $\theta$ between adjoining blocks (and adjacent quadrants).

From "engineering" constraints (see §IIB and IIC discussion), the iron yokes of adjoining magnets must be separated approximately $13 \mathrm{~cm}$ in $z$. We have also specified that each end of the yoke should extend approximately one wire radius $(8.5 \mathrm{~cm})$ in $z$ beyond the outermost coil wire in order to confine the fringe fields in $z$. Hence, the maximum $z$-length of each coil is thus $\approx 30 \mathrm{~cm}$ for $l=60 \mathrm{~cm}$. Setting the $z$-extent $(2.0 \mathrm{~cm})$ of the curve of each coil block end, the $z$-separation $(1.0 \mathrm{~cm})$ between adjoining blocks, and the wire diameter $(0.32 \mathrm{~cm}$ for our particular braided copper choice), the total $z$ length of each block and its individual wires is fully determined. The azimuthal positions of each block are then found numerically by forcing the $z$-integrated harmonic overtones to zero (see ref. [3] for details).

In our previous prototype which had been constructed primarily to investigate thermal heat dissipation, we had not paid careful attention to either the geometry of the wire crossovers from one block to another (which caused net dipole current loops) nor the presence of a solenoidal loop caused by the azimuthal advance of the wire leads leaving one quadrant and entering another. In our current design, we have carefully created " $\mathrm{X}$ "-shaped crossovers (see Fig. 1) when projected on the $\theta-z$ plane whose net dipole area is less than $0.5 \mathrm{~cm}^{2}$ for each layer of each quadrant. We have also returned the exiting wire lead of the fourth quadrant back the "long way" azimuthally to the first quadrant in order to eliminate the solenoidal loop problem. Hence, we expect a much higher quality magnetic field when we make detailed measurements of this magnet over the next year.

We have done a series of POISSON and TOSCA calculations to examine the effects of the iron yoke upon the magnetic field. The 2D POISSON runs (Fig. 2 shows the output from one example) suggest that at field gradients $\leq 26.5 \mathrm{~T} / \mathrm{m}$ the maximum B-field strength in the yoke remains below $1.5 \mathrm{~T}$ and saturation effects are minimal. We are particularly concerned with saturation (which will be due to the dominant quadrupole component) destroying the $z$-integrated cancellation of higher harmonics (in particular the dodecapole) within each half-lattice period. The 3D TOSCA runs indicate that field gradients of up to $28.6 \mathrm{~T} / \mathrm{m}$ at the magnet center in $z$ are possible with minimal field leakage $(\leq 10 \mathrm{G})$ radially outside the iron yoke. When the $95 \%$ packing fraction of the iron laminations is included in the TOSCA runs, field gradients of up to $26 \mathrm{~T} / \mathrm{m}$ appear to be safe. Both codes show that the yoke enhances the interior quadrupole field gradient by approximately $20 \%$. The effective length of the quadrupole component is $0.24 \mathrm{~m}$; without enhancement from the iron yoke, a field gradient of $19.5 \mathrm{~T} / \mathrm{m}$ (corresponding to $4.2 \mathrm{kA}$ wire current) is required for $\sigma_{0}=72^{\circ}$ at $5 \mathrm{MV}$.

\section{$B$. Iron Yoke Design}

The iron yoke enclosing the current windings and the beam pipe serves a number of purposes. First, it minimizes magnetic fringe fields from extending greatly in $z$ or $r$ and interfering either with the beam transport or the induction core material (and visa versa). It also mechanically confines the windings and prevents their expansion and distortion under the magnetic stresses. Finally, it also provides a fiducial for mechanical alignment and adjustment.

In designing the yoke, the two major requirements were first to make sure that we had a sufficient amount of iron at a great enough distance from the conductors such that the iron will not saturate during pulsed operation, thus adversely effecting the quality of the magnetic field, and 


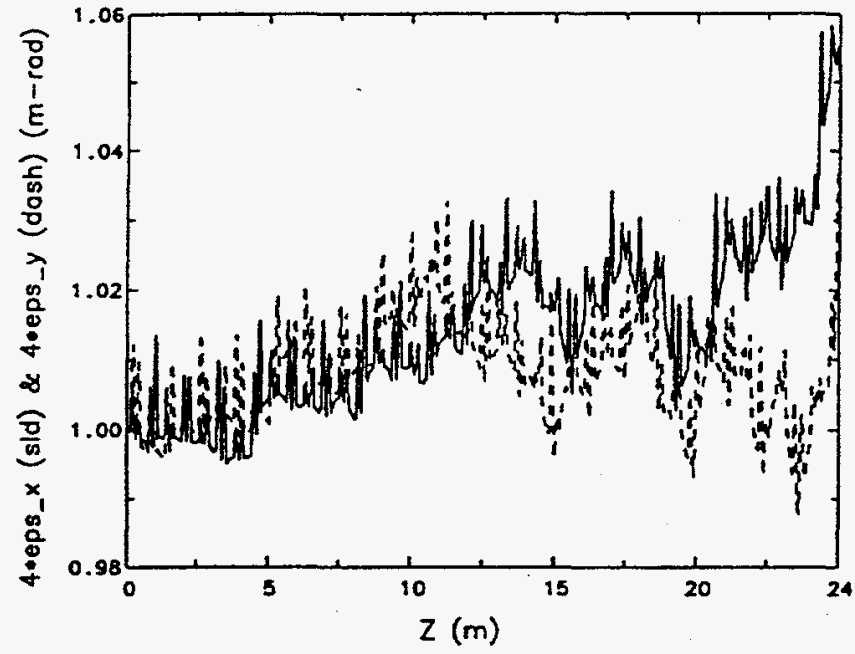

Figure. 3. HIFI simulation code results for emittance growth, normalized to the initial value of $\varepsilon_{N}=5 \pi \mathrm{mm}$ mrad, over $24 \mathrm{~m}$ of magnetic transport of a $1.5 \mu \mathrm{C} / \mathrm{m} \mathrm{K}^{+1}$ beam at $\sigma_{0}=72^{\circ}$ and $\sigma=7^{\circ}$. The solid curve refers to the $x-x^{\prime}$ plane and the dashed curve to the $y-y^{\prime}$ plane.

secondly to minimize the effective outer diameter of the yoke once the inner diameter had been fixed due to the magnetic saturation concerns.

Minimizing the effective outer diameter of the yoke reduces the weight, making suport and articulation easier, but, more importantly, it also reduces the inner diameter of the induction cores which surround the magnet. The annular shaped induction cores have a fixed, required cross sectional area. Hence, choosing a larger I.D. for the cores leads to greater volume and cost. In order to minimize the effective diameter in our second prototype, we changed the material of the yoke to low carbon steel which has a higher saturation induction of $2.08 \mathrm{~T}$ as compared to $1.96 \mathrm{~T}$ for the $3 \%$ silicon steel used in a prototype previously constructed. We also changed the originally square cross section of the yoke to an irregular octagon by cutting off the "corners" of the square yoke and checking for saturation effects with the POISSON code. We chose an inner yoke radius of $12.13 \mathrm{~cm}$ which is large enough to allow the magnetic fields to fall off by more than a factor of two from their values at the current windings but not so large as to increase magnetic core costs significantly. Taken together, these modifications reduced the effective outer yoke radius by $3 \mathrm{~cm}$ which translates into a projected savings of $\approx 250 \mathrm{~K} \$$ on the magnetic quadrupole focusing section for ILSE.

\section{Mechanical Design Details}

In order that the magnets be powered, have their heat dissipated safely, and the beam actually be accelerated, a number of elements must be placed between adjoining magnets. In particular, electrical insulators made of $98 \%$ alumina with field grading rings are necessary to prevent. electrical breakdown in the accelerating fields produced by the induction cores. These insulators occupy $\approx 80 \%$ of the required intermagnet gap. The gap also includes $O$ ringed flanges and bellows to support the insulators ant magnets, water pipes for the required magnet cooling water $(\sim 3 \mathrm{GPM})$, and insulated electrical leads $(10 \mathrm{kV}$ required for $5.4 \mathrm{kA}$ current). Fortunately, many of the components can be made re-entrant with the iron yoke to reduce the required intermagent separation.

\section{Particle Simulation Code Studies}

We have used the 2D particle simulation code "HIFI" [3] to model the transport dynamics of a $1.5 \mu \mathrm{C} / \mathrm{m}$ spacecharge dominated 5-MV, $\mathrm{K}^{+1}$ beam through $24 \mathrm{~m}$ of magnetic transport without acceleration gaps. The magnetic fields, comprised of the quadrupole, dodecapole, pseudooctupole, and pseudo-dodecapole components, were taken from a 3D field solution which does not include the effects of the iron yoke. The peak field gradient at the center of each magnet was set to $19.5 \mathrm{~T} / \mathrm{m}$ giving an undepressed phase advance $\sigma_{0}$ of 72 degrees per lattice period. The normalized edge emittance of the beam was set to $5 \pi \mathrm{mm}$ mrad which gave to a space-charge depressed tune $\sigma$ of approximately 7 degrees. The beam was initialized with a $\mathrm{KV}$ distribution in transverse phase space which led to a maximum beam envelope extent of $69 \mathrm{~mm}$ as compared with a presumed beam pipe radius of $75 \mathrm{~mm}$. Over the 20 lattice periods, no simulation particles were lost and the emittance growth was of order $5 \%$ or less. Consequently, we are confident that if the magnet performs according to design it will be able to transport the specified beam line charge without serious degradation.

\section{References}

[1] Laslett, L.J., Caspi, S., and Helm, M., Particle Accel., 22, 1 (1987).

[2] Fawley, W.M., Laslett, L.J., Celata, C.M., Faltens, A., and Haber, I., "Simulation Studies of Space-ChargeDominated Beam Transport in Large Aperture Ratio Quadrupoles", Proceedings of the 1999 IEEE Particle Accelerator Conference, 93CH3279-7, 724 (1993).

[3] Fawley, W.M., "Space-dominated Beam Transport -in Magnetic Quadrupoles with Large Aperture Ratios", LBL-33608, 1993. 